\title{
THE SUBNORMAL COALESCENCE OF SOME CLASSES OF GROUPS OF FINITE RANK
}

\author{
Dedicated to the memory of Hanna Neumann
}

\author{
MARK DRUKKER, DEREK J. S. ROBINSON and IAN STEWART
}

(Received 13 June 1972)

Communicated by M. F. Newman

A class $\mathfrak{X}$ of groups forms a (subnormal) coalition class, or is (subnormally) coalescent, if whenever $H$ and $K$ are subnormal $\mathfrak{X}$-subgroups of a group $G$ then their join $\langle H, K\rangle$ is also a subnormal $\mathfrak{X}$-subgroup of $G$. Among the known coalition classes are those of finite groups and polycyclic groups (Wielandt [15]); groups with maximal condition for subgroups (Baer [1]); finitely generated nilpotent groups (Baer [2]); groups with maximal or minimal condition on subnormal subgroups (Robinson [8], Roseblade [11,12]); minimax groups (Roseblade, unpublished); and any subjunctive class of finitely generated groups (Roseblade and Stonehewer [13]).

A group $G$ has finite rank $r$, for a positive integer $r$, if every finitely generated subgroup of $G$ can be generated by $r$ elements and if $r$ is the least such integer. (This is the Mal'cev special rank, cf. Kuroš [5]). We shall prove that the class of all groups of finite rank, and certain of its subclasses (in particular nilpotent, soluble, locally nilpotent, and locally soluble groups of finite rank) are coalition classes.

Our notation will be consistent with that of Robinson [9]. In particular if $H$ is a subgroup of $G$ and $n$ is a positive integer then $H^{G, n}$ is the $n$th term of the normal closure series of $H$ in $G$. The group $[H, K]$ is that generated by all commutators $h^{-1} k^{-1} h k$ for $h \in H, k \in K$; and inductively we define

$$
\left[H,{ }_{n+1} K\right]=\left[\left[H,{ }_{n} K\right], K\right] \text {. }
$$

The closure operations $\mathrm{L}$ and $\mathrm{s}_{n}$ are defined as follows: for any class $\mathfrak{X}$ of groups, $s_{n} \mathfrak{X}$ consists of all subnormal subgroups of $\mathfrak{X}$-groups. A group $G$ is in $L \mathfrak{X}$ if every finite subset of $G$ is contained in an $\mathfrak{X}$-subgroup of $G ; \mathfrak{L} \mathfrak{X}$ is the class of locally- $\mathfrak{X}$ groups.

We may now state our main theorem. 
THEOREM 1. Let $\mathfrak{X}_{1}, \mathfrak{X}_{2}, \cdots$ be a sequence of $\mathrm{L}-$ and $\mathrm{s}_{n}$-closed classes of groups, and let

$$
\mathfrak{X}=\bigcup_{i=1}^{\infty} \mathfrak{X}_{i} \text {. }
$$

Assume that the product of a normal $\mathfrak{X}_{i}$-subgroup and a normal $\mathfrak{X}_{j}$-subgroup always belongs to $\mathfrak{X}_{k}$ where $k=k(i, j)$. Then the class of $\mathfrak{X}$-groups of finite rank is a subnormal coalition class.

COROLLARY. Groups of finite rank, soluble groups of finite rank, and nilpotent groups of finite rank form subnormal coalition classes.

Proof. For the first case, take each $\mathfrak{X}_{i}$ to be the class of all groups; for the second the class of soluble groups of derived length $\leqq i$; for the third the class of nilpotent groups with class $\leqq i$.

(Of course, the results of Stonehewer [14] show that two subnormal soluble groups always generate a soluble group.) The theorem will follow from three standard lemmas.

LEMMA 1. If $H \leqq G$ and $X$ is an s-generator subgroup of $G$, then

$$
H^{X}=\left\langle H_{1},\left[H,{ }_{v} X\right]\right\rangle
$$

for any $v>0$, where $H_{1}$ is generated by at most $1+s+\cdots+s^{v-1}$ conjugates of $H$ in $X$.

Proof. Robinson [9] Lemma 3.21. See also Roseblade and Stonehewer [13].

LEMMA 2. If $N \triangleleft G$ and $N$ has finite rank $r$ and $G / N$ has finite rank $r^{\prime}$, then $G$ has finite rank $\leqq r+r^{\prime}$.

Proof. Baer [3] Lemma 1.3.

Lemma 3. Let $\mathfrak{X}_{1}, \mathfrak{X}_{2}, \cdots$ be a sequence of $s_{n}$-closed classes such that the condition on normal products in the theorem is satisfied. Let $H \triangleleft^{\prime \prime} G, K \triangleleft^{v} G$, $H^{K}=H$ and suppose that $H \in \mathfrak{X}_{i}$ and $K \in \mathfrak{X}_{j}$. If $J=<H, K>$ then $J \triangleleft^{\prime \prime v} G$ and $J \in \mathfrak{X}_{l}$ where $l=l(i, j, v)$.

Proof. As in Robinson [9] Lemma 3.15.

Proof of Theorem. Let $H \triangleleft^{u} G, K \triangleleft^{v} G$ and suppose that $H$ and $K$ have finite ranks $r$ and $s$ respectively. Let $H \in \mathfrak{X}_{i}$ and $K \in \mathfrak{X}_{j}$. Write $J=\langle H, K\rangle$. We shall prove that $J \in \mathfrak{X}_{f}, J$ has finite rank $\leqq g$, and $J \triangleleft^{h} G$ where $f, g, h$ are integers $\geqq 0$ depending only on $i, j, r, s, u, v$. If $u \leqq 1$, then $H \triangleleft G$ and the result follows from Lemmas 2 and 3. Therefore let $u>1$ and $v>1$.

Let $X$ be any finitely generated subgroup of $K$; then $X$ is an $s$-generator group and

$$
H^{X}=\left\langle H_{1},[H, X]\right\rangle
$$


where $H_{1}$ is generated by $1+s+\cdots+s^{v-1}$ conjugates of $H$ in $X$ by lemma 1 . Write

$$
H(X)=\left\langle H^{X},\left[H,{ }_{0} K\right]\right\rangle=\left\langle H_{1},\left[H,{ }_{v} K\right]\right\rangle .
$$

From $K \triangleleft^{v} G$ it follows that $\left[H,{ }_{v} K\right] \sqsupset K$; thus $\left[H,{ }_{v} K\right] \in \mathfrak{X}_{j},\left[H,{ }_{v} K\right]$ has rank $\leqq s$ and $\left[H,{ }_{v} K\right] \triangleleft^{v+1} G$. Also $H^{v} \triangleleft^{u+1} H^{g}$ if $s \in X$. Therefore we can use induction on $u$ to show that $H(X) \in \mathfrak{X}_{f^{\prime}}, H(X)$ has finite rank $\leqq g^{\prime}$ and $H(X) \triangleleft^{h^{\prime}} G$ where $f^{\prime}, g^{\prime}, h^{\prime}$ depend only on $i, j, r, s, u, v$. Now obviously any two $H(X)$ 's are contained in a third, and

$$
H^{K}=\bigcup H(X),
$$

the union being over all finitely generated subgroups $X$ of $K$. From this it is clear that $H^{K} \in \mathrm{L} \mathfrak{X}_{f^{\prime}}=\mathfrak{X}_{f^{\prime}}$ and $H^{K}$ has rank $\leqq g^{\prime}$. Let $y \in\left(H^{K}\right)^{G, h^{\prime}}$, the $h^{\prime}$-th normal closure of $H^{K}$ in $G$. Clearly $y \in(H(X))^{G \cdot h^{\prime}}=H(X) \leqq H^{K}$ for some finitely generated subgroup $X$ of $K$. Therefore $\left(H^{K}\right)^{G, h^{\prime}}=H^{K}$ and $H^{K} \triangleleft^{h^{\prime}} G$.

Finally, $J=H^{K} K$ and the result follows from lemmas 2 and 3.

We may now fill in some extra detail, as follows:

THEOREM 2. Locally nilpotent groups of finite rank and locally soluble groups of finite rank form coalition classes.

Proof. The first assertion follows immediately from Theorem 1 and the Hirsch-Plotkin theorem (Hirsch [4], Plotkin [7]). It should be noted that for groups of finite rank local nilpotence is equivalent to hypercentrality, from resu.ts of Mal'cev [6].

For the second assertion, let $H$ and $K$ be subnormal in $G$, each locally soluble of finite rank, and let $J=\langle H, K\rangle$. By Robinson [10] $H^{(n)}$ is hypercentral for some $n$, so that the upper Hirsch-Plotkin series (of iterated Hirsch-Plotkin radicals) of $H$ reaches $H$ in finitely many steps. Similarly for $K$. The Hirsch-Plotkin theorem shows that the upper Hirsch-Plotkin series of $J$ reaches $J$ in finitely many steps. We know that $J$ has finite rank; that it is also locally soluble follows from the main theorem of Robinson [10]. Of course $J$ sn $G$ by theorem 1, and its corollary.

It should be noted that the class of locally soluble groups is not even $\mathrm{N}_{0}$-closed (where $\mathfrak{X}$ is $N_{0}$-closed if the join of two normal $\mathfrak{X}$-subgroups is in $\mathfrak{X}$ ) as is shown by a famous example of Hall (Robinson [9] theorem 4.24). Further, locally nilpotent groups of finite rank need not be soluble; the relevant example being due to Kegel (see Baer [3] p.27) and consisting of a direct product of finite $p$-groups, for different $p$, having bounded rank but unbounded derived length.

It is easy to see that nilpotent groups of finite rank do not form an ascendant coalition class (defined in the obvious way), by considering the split extension of a group of type $C_{2} \infty$ by the automorphism which inverts every element. We have not decided whether groups of finite rank, or soluble groups of finite rank, form an ascendant coalition class. 


\section{References}

[1] R. Baer, 'Lokal Noethersche Gruppen', Math. Z. 66 (1957), 341-363.

[2] R. Baer, 'Nil-Gruppen', Math. Z. 62 (1955), 402-437.

[3] R. Baer, 'Polyminimaxgruppen', Math. Ann. 175 (1968), 1-43.

[4] K. A. Hirsch, 'Uber lokal-nilpotente Gruppen', Math. Z. 63 (1955), 290-294.

[5] A. G. Kuroš, Theory of groups vol. 2, (Chelsea, New York, 1956). Translated by K. A. Hirsch.

[6] A. I. Mal'cev, 'On certain classes of infinite soluble groups', Mat. Sb. (N. S.) 28 (70) (1951), 567-588. Amer. Math. Soc. Translations (2) 2 (1956), 1-21.

[7] B. I. Plotkin, 'On some criteria of locally nilpotent groups', Uspehi Mat. Nauk (N.S.) 9 (1954), 181-186. Amer. Math. Soc. Translations (2) 17 (1961), 1-8.

[8] D. J. S. Robinson, 'On the theory of subnormal subgroups', Math, Z. 89 (1965), 30-51.

[9] D. J. S. Robinson, Infinite soluble and nilpotent groups, (QMC Mathematics Notes, London 1968).

[10] D. J. S. Robinson 'A note on groups of finite rank', Compositio Math. 21 (1969), 240-246.

[11] J. E. Roseblade, 'On certain subnormal coalition classes', J. Algebra 1 (1964), 132-138.

[12] J. E. Roseblade, 'A note on subnormal coalition classes', Math. Z. 90 (1965), 373-375.

[13] J. E. Roseblade and S. E. Stonehewer, 'Subjunctive and locally coalescent classes', J. Algebra 8 (1968), 423-435.

[14] S. E. Stonehewer, 'The join of finitely many subnormal subgroups', Bull. London. Math. Soc. 2 (1970), 77-82.

[15] H. Wielandt, 'Eine Verallgemeinerung der invarianten Untergruppen', Math. Z. 45 (1939), 209-244.

University of Warwick

Coventry, Warwickshire CV4, 7AL

England

University of Illinois

Urbana, Illinois 61801 U.S.A. 